\title{
IGHG1 induces EMT in gastric cancer cells by regulating TGF-B/SMAD3 signaling pathway
}

\author{
Yuxuan $\mathrm{Li}^{1 *}$, Pan Wang ${ }^{2^{*}}$, Dongmei Ye ${ }^{3}$, Xue Bai ${ }^{1}$, Xuemei Zeng ${ }^{1}$, Qiang Zhao ${ }^{4}$, Zhiwei Zhang ${ }^{\circledR}$ \\ 1. Key Laboratory of Cancer Cellular and Molecular Pathology in Hunan Province, Cancer Research Institute of Hengyang Medical College, University of \\ South China, Hengyang, 421001, Hunan Province, China. \\ 2. Department of Pathology, Qingyuan People's Hospital, Qingyuan, 511500, Guangdong Province, China. \\ 3. Department of Pathology, The Third Affiliated Hospital of Nanchang University, Nanchang, 330008, Jiangxi Province, China. \\ 4. Department of Pathology, The First Affiliated Hospital of University of South China, Hengyang, 421001, Hunan Province, China. \\ *These authors contributed equally to this work.
}

$\square$ Corresponding author: Zhiwei Zhang, Cancer Research Institute of Hengyang Medical College, University of South China; Key Laboratory of Cancer Cellular and Molecular Pathology in Hunan Province, 28 Changsheng Road, Hengyang, 421001, Hunan, People's Republic of China; Tel: 13975497643, E-mail: nhdxzzw@qq.com, Fax: 86-734-8281075.

(c) The author(s). This is an open access article distributed under the terms of the Creative Commons Attribution License (https://creativecommons.org/licenses/by/4.0/). See http://ivyspring.com/terms for full terms and conditions.

Received: 2020.11.17; Accepted: 2021.03.29; Published: 2021.04.19

\begin{abstract}
Objective: Gastric cancer is one of the most common malignant tumors in the world. IGHGl is a differentially expressed protein screened out in gastric cancer in the early stage of the subject group. This topic explores the expression of IGHGI in gastric cancer and the effect of IGHGI on the proliferation, migration, invasion and EMT of gastric cancer SGC7901 cells and its mechanism of action.

Methods: Twenty cases of gastric cancer were purified by laser Capture Microdissection. The isotopic tags for relative and absolute quantification was used to label the proteins, and then analyzed and identified them by quantitative proteomics. Immunohistochemical staining method was used to detect the expression of IGHGI protein in gastric cancer tissues. Western blot was used to detect the expression of IGHGI in gastric cancer cells. The MTT and Petri dish clone formation experiment analyzed the effect of low expression of IGHGI on the proliferation of SGC790I cells. Scratch test and Transwell migration and invasion test to observe the effect of low expression of IGHGI on the migration and invasion of SGC7901 cells. Western blot was used to detect the effect of low expression of IGHGI on the expression of EMT-related proteins.
\end{abstract}

Results: 243 proteins related to gastric mucosal lesions were preliminarily identified. We found that IGHGI is highly expressed in gastric cancer tissues compared with normal control tissues. IGHGI promotes the proliferation, migration and invasion of gastric cancer cells. Compared with the control group, the expression of EMT-related proteins Vimentin, N-cadherin, TGF- $\beta$, P-SMAD3 was decreased and the expression of E-cadherin was increased after IGHGI low expression.

Conclusions: IGHGI induces EMT in SGC7901 cells by regulating the TGF- $\beta / S M A D 3$ signaling pathway.

Key words: gastric cancer; IGHG1; migration and invasion; EMT TGF- $\beta$ /SMAD signaling pathway

\section{Introduction}

Gastric cancer (GC) is one of the most common malignant tumors in the world. The 2018 global cancer statistics show that from 2015 to 2018, there were more than 1 million new cases of gastric cancer and 783,000 deaths, ranking the fifth in the global incidence of malignant tumors and the third in the mortality rate of malignant tumors [1]. The incidence of gastric cancer has gender differences, which the incidence of men is twice that of women. However, due to the degree of economic development and the difference in society and lifestyle, there are also obvious differences between different regions and different countries. In China, gastric cancer is one of the most common malignant tumors, and its morbidity and mortality rank second among all malignant tumors. The incidence of males is higher 
than that of females, and the age of onset of gastric cancer is mainly concentrated in 45-75 years old [2], which seriously endangering people's health and lives.

The IGHG1 gene is located on chromosome 14 q32.33, and the protein encoded by it is one of the subtypes of immunoglobulin IgG, which is located in the $C$ region of the Ig -1 chain [3]. There are five different types of human immunoglobulins: $\operatorname{IgM}, \operatorname{IgD}$, IgG, IgE, and IgA [4]. IgG is an important component of the adaptive immune system, accounting for $75-80 \%$ of the total Ig pool [5]. IgG is composed of heavy and light chains, including a chain, $\delta$ chain, $\varepsilon$ chain, $\gamma$ chain and $\mu$ chain, and light chains including $\kappa$ chain and $\lambda$ chain. IGHG1 plays an important role in the occurrence and development of tumors. Its expression in ovarian cancer [6,7], pancreatic cancer [3], prostate cancer [8,9], papillary thyroid carcinoma [5] and other malignant epithelial tumors is significantly increased. It is involved in the pathological process of tumor cell EMT, proliferation, apoptosis resistance, immune escape and metastasis.

\section{Materials and methods}

\section{Tissue samples}

For LCM, 20 paired samples of normal gastric mucosa, atypical hyperplasia, poorly differentiated gastric adenocarcinoma, and lymph node metastatic carcinoma were taken from surgical resection of tumor specimens in the First Affiliated Hospital of South China University. None of the above patients received preoperative radiotherapy and chemotherapy.

\section{Laser capture microdissection}

The target tissue was captured from frozen sections of gastric mucosal lesions using a Laser Capture Microdissection Microscope. Adjust the laser aperture, speed and intensity according to the distribution of field of vision, magnification and target tissue. In order to reduce individual genetic differences, all cut and purified tissues were from different tissues of 20 patients with gastric cancer. The purified tissues were classified and mixed for identification by iTRAQ labeling and twodimensional liquid chromatography coupled with mass spectrometry.

\section{iTRAD labeling and LC-MS/MS analysis}

Purified protein sample processed according to the manufacturer's protocol for iTRAQ reagent (Applied Biosystems). Dissolve the sample to be tested in $1 \mathrm{ml} \mathrm{SCX}$ buffer $(25 \% \mathrm{v} / \mathrm{v}$ acetonitrile, 10 $\mathrm{mM} \mathrm{KH} \mathrm{PO}_{4}$, $\mathrm{pH}$ 2.6), insert the sample solution to be tested into Polysulfoethyl column $(2.1 \mathrm{~mm} \times 100 \mathrm{~mm}, 5$ 4m, $200 \AA$, The Nest Group, Inc.MA), Separation by 20 AD HPLC systematic chromatography, vacuum centrifugal concentration, dissolving with $50 \mu \mathrm{L}$ RPLC A (5\% CAN, $0.1 \%$ formic acid). The mixture was then separated on a $\mathrm{C}_{18}$ reverse-phase column (Zorbax $300 S \mathrm{~N}-\mathrm{C}_{18}, 0.1 \times 15 \mathrm{~mm}, 5 \mu \mathrm{m}, 300 \AA, 4.6 \times 250 \mathrm{~mm}$; microm, USA) at a flow rate of $300 \mu \mathrm{L} / \mathrm{min}$ for $90 \mathrm{~min}$. he q-Star XL(Applied Biosystems, USA) system was used for MS/MS analysis.

ProteinPilot ${ }^{\mathrm{TM}}$ software (Version 4.2, revision 1340) was used for database search. The mass spectrum data analyzed by Analyst QS1.1 (Applied Biosystems) is imported into ProteinPilot ${ }^{\mathrm{TM}}$, choose Paragon Method for analysis.

\section{Case data}

The tissue chip of gastric cancer was presented by Mrs. Xia Hong from the Cancer Institute of Nanhua University. Tissue microarray samples were collected from 90 cases of gastric cancer patients, including 20 highly differentiated adenocarcinoma tissues, 29 moderately differentiated adenocarcinoma tissues, 21 poorly differentiated adenocarcinoma tissues, 9 signet ring cell tissues, and 11 mucinous adenocarcinoma tissues. 70 normal gastric mucosa tissues from 90 gastric cancer (taken from normal gastric mucosa above $10 \mathrm{~cm}$ from the edge of the tumor). None of the above patients received radiotherapy or chemotherapy before surgery, and the diagnosis was confirmed by the pathologist.

\section{Cell line}

Human immortalized gastric mucosal epithelial GES-1 cell line, human gastric cancer MGC803 cells, BGC823 cells and SGC7901 cell line were provided by the Tumor Research Institute of Nanhua University.

All the cell lines were cultured in a complete medium containing a mixture of 1640 medium (RPMI) and fetal bovine serum at a ratio of 9:1, and the culture flask was placed in a constant temperature and humidity cell incubator for routine culture.

\section{Antibodies and reagents used in the experiment}

IGHG1 antibody was purchased from Abnova. TGF- $\beta$ was purchased from Abcam, UK.E-cadherin, Vimentin, N-cadherin, SMAD3 and P-SMAD3 were purchased from CST USA. $\beta$-actin was purchased from Biosharp. Fluorescent secondary antibodies were purchased from LI-COR, USA. The IGHG1 interference carrier was purchased from PLL (Changsha Jiahe Biotechnology Co., Ltd.). RPMI 1640 cell culture medium and trypsin were purchased from Gibco, USA. The ready-to-use immunohistochemistry kit was purchased from Fuzhou Maixin Biotechnology Development Co., Ltd. DAB dyeing 
liquid was purchased from Kangwei Century Biotechnology Co., Ltd. Matrigel adhesive was purchased from Corning, USA.

\section{Immunohistochemistry (IHC)}

The gastric cancer tissue chip was dewaxed to water and repaired with citric acid $(\mathrm{P}=6.0)$. Immunohistochemical staining was performed with reference to the instructions of the two-step assay kit (KIT-9710, Fuzhou Maixin Biotechnology Development Co., Ltd.). The IGHG1 antibody was diluted with PBS buffer at a dilution ratio of 5:50, DAB was developed, and hematoxylin was stained with nuclei.

\section{Plasmids and transfection}

Three IGHG1 interference vectors were constructed. The target sequence information was CCAAGGACACCCTCATGAT and AGTGCAAGGT CTCCAACAA and GCTCCTTCTTCCTCTACAG, respectively, and the sequencing confirmed that the sequence is correct. The LipoMax transfection reagent transiently transfected the interference vector into SGC7901 cells.

\section{Immunoblot assay}

Total cellular protein was extracted using a lysis buffer containing a protease inhibitor. The protein concentration was determined by the BCA method. Total protein was separated on an SDS-PAGE gel and transferred to a PVDF membrane. Skim milk is blocked and antibody-fed, using $\beta$-actin as an internal reference.

\section{Proliferation assay}

Inoculate 5000 cells per well into 96 -well plates, set 3-5 replicate wells, add $20 \mu \mathrm{L}(5 \mathrm{mg} / \mathrm{mL})$ of MTT solution to each well at $0 \mathrm{~h}, 24 \mathrm{~h}, 48 \mathrm{~h}, 72 \mathrm{~h}$ and $96 \mathrm{~h}$ after inoculation, avoiding light in the incubator Continue to culture for 4 hours. $150 \mu \mathrm{L}$ of DMSO was added to each well, and the mixture was shaken for 10 $\min$ at a constant temperature oscillator $\left(37^{\circ} \mathrm{C}, 200\right.$ $\mathrm{rpm})$, and a single wavelength of $570 \mathrm{~nm}$ was selected using a microplate reader to measure the absorbance.

\section{Colony formation assay}

800 cells per well were inoculated into a 6-well plate, and a secondary well was set, and the culture solution was periodically replaced. When the cell mass was visible to the naked eye, the culture was terminated, the paraformaldehyde solution was fixed, and the crystal violet staining solution was stained, and the microscopic count was greater than 50 cell clones.

\section{Scratch assay}

$4-6 \times 10^{5}$ cells per well were seeded in 6-well plates. When the degree of cell fusion reaches $90 \%$, use $200 \mu \mathrm{L}$ Tip head to scratch $1 / 4,1 / 2$ and $3 / 4$ of the cell surface of the pore plate with a straight ruler. Take a picture and record the distance of the scratches at $0 \mathrm{~h}, 24 \mathrm{~h}$ and $48 \mathrm{~h}$ after the scratch.

\section{Transwell migration invasion assay}

Matrigel and RPMI were formulated into working fluids in a ratio of 1:6. $50 \mu \mathrm{l}$ of the working solution was added to the upper chamber of each chamber, evenly tiling, and incubated for $7 \mathrm{~h}$ (this step is not required for the migration assay). $200 \mu \mathrm{l}$ (density $2 \times 10^{5}$ cells $/ \mathrm{mL}$ ) cell suspension was added to the Matrigel-coated Transwell chamber. Fixed with paraformaldehyde $24 \mathrm{~h}$ later, stained with crystal violet staining solution. The number of invading cells was counted by three fields under the microscope.

\section{Statistical analysis}

This study used paswstat and GraphPad Prism 5 software for statistical analysis. The data listed in this study are expressed as mean \pm standard deviation, in which immunohistochemical staining results are statistically analyzed using Fisher's exact probability test; Western blot and cell clone formation assay results were analyzed by One-way ANOVA; MTT assay results were analyzed by Two-way ANOVA. All statistical analysis results showed that the difference was statistically significant at $P<0.05$.

\section{Results}

\section{The differentially expressed proteins in different stages of gastric mucosa canceration were screened}

In order to further understand the molecular mechanism of gastric cancer and obtain valuable diagnostic markers. The research group collected twenty matched gastric cancer tissues classified as normal gastric mucosa (NGM), atypical hyperplasia $(\mathrm{AH})$, gastric poorly differentiated adenocarcinoma (GPDAC) and lymph nodes metastasis adenocarcinoma (LMGAC) for surgical excision of specimens. The above tissues were purified by Laser capture microdissection (LCM). Total proteins in different tissues were extracted and isotopically labeled. Through isobaric tags for relative and absolute quantification (iTRAQ) combined with two-dimensional liquid chromatography-tandem mass spectrometry (2D LC-MS/MS), the analytical chromatography of these different tissues was performed for quantitative analysis and detection. The expression of the identified protein was 
up-regulated when the quantitative ratio was greater than 1.5 and down-regulated when the quantitative ratio was less than 0.667 . A total of 243 proteins related to gastric epithelial cancer were identified, 153 of which were up-regulated (Table 1) and 90 downregulated (Table 2) in gastric cancer tissues. Among them, IGHG1 was up-regulated in gastric cancer.

Table 1. Quantitative proteomics to identify up-regulated proteins in different stages of gastric mucosal epithelial carcinoma

\begin{tabular}{|c|c|c|c|c|c|}
\hline No & Protein Name & $\begin{array}{l}\text { AH vs. } \\
\text { NGM }\end{array}$ & $\begin{array}{l}\text { GPDAC } \\
\text { vs. NGM }\end{array}$ & $\begin{array}{l}\text { LMGAC } \\
\text { vs. NGM }\end{array}$ & $\begin{array}{l}\text { LMGAC } \\
\text { vs. } \\
\text { GPDAC }\end{array}$ \\
\hline 1 & DDOST & $\uparrow 2.559$ & $\uparrow 1.803$ & $\uparrow 2.831$ & $\uparrow 8.017$ \\
\hline$\underline{2}$ & $\underline{\text { IGHG1 protein }}$ & $\uparrow 1.690$ & $\uparrow 2.421$ & $\uparrow 4.245$ & $\uparrow 2.291$ \\
\hline 3 & $\begin{array}{l}\text { GPI Glucose-6-phosphate } \\
\text { isomerase }\end{array}$ & $\uparrow 2.673$ & $\uparrow 2.805$ & $\uparrow 1.677$ & $\uparrow 9.201$ \\
\hline 4 & $\begin{array}{l}\text { PPIB peptidylprolyl isomerase } \\
\text { B precursor }\end{array}$ & $\uparrow 2.489$ & $\uparrow 4.325$ & $\uparrow 1.820$ & $\uparrow 2.377$ \\
\hline 5 & PRSS1 protein & $\uparrow 4.966$ & $\uparrow 4.446$ & $\uparrow 7.253$ & $\uparrow 3.076$ \\
\hline 6 & RTN4 Isoform 1 of Reticulon- 4 & $\uparrow 3.221$ & $\uparrow 4.529$ & $\uparrow 3.253$ & $\uparrow 16.596$ \\
\hline 7 & LGALS3 Galectin-3 & $\uparrow 7.112$ & $\uparrow 6.918$ & $\uparrow 2.466$ & $\uparrow 2.805$ \\
\hline 8 & Heat shock protein HSP 90 & $\uparrow 3.664$ & $\uparrow 7.379$ & $\uparrow 3.311$ & $\uparrow 2.228$ \\
\hline 9 & Myosin-9 & $\uparrow 4.525$ & $\uparrow 7.586$ & $\uparrow 3.342$ & $\uparrow 2.270$ \\
\hline$\ldots$ & $\ldots$ & $\ldots$ & $\ldots$ & $\ldots$ & $\ldots$ \\
\hline 145 & TGM2 & $\uparrow 7.112$ & $\uparrow 7.870$ & $\uparrow 3.631$ & $\uparrow 2.168$ \\
\hline 146 & Secernin-1 & $\uparrow 1.959$ & $\uparrow 7.870$ & $\uparrow 1.660$ & $\uparrow 4.742$ \\
\hline 147 & $\begin{array}{l}\text { HSP90B1 Endoplasmin } \\
\text { precursor }\end{array}$ & $\uparrow 4.742$ & $\uparrow 8.395$ & $\uparrow 1.995$ & $\uparrow 4.207$ \\
\hline 148 & type I cytoskeletal 17 & $\uparrow 2.228$ & $\uparrow 8.710$ & $\uparrow 2.333$ & $\uparrow 3.733$ \\
\hline 149 & Biglycan precursor & $\uparrow 4.790$ & $\uparrow 10.093$ & $\uparrow 3.945$ & $\uparrow 2.559$ \\
\hline 150 & type I cytoskeletal 19 & $\uparrow 5.248$ & $\uparrow 11.482$ & $\uparrow 4.446$ & $\uparrow 2.582$ \\
\hline 151 & $\begin{array}{l}\text { COMP Cartilage oligomeric } \\
\text { matrix protein precursor }\end{array}$ & $\uparrow 1.660$ & $\uparrow 12.023$ & $\uparrow 3.192$ & $\uparrow 3.767$ \\
\hline 152 & Cathepsin $\mathrm{Z}$ precursor & $\uparrow 8.551$ & $\uparrow 12.706$ & $\uparrow 6.546$ & $\uparrow 1.941$ \\
\hline 153 & $\begin{array}{l}\text { Collagen alpha-1(XII) chain } \\
\text { precursor }\end{array}$ & $\uparrow 4.267$ & $\uparrow 14.997$ & $\uparrow 4.366$ & $\uparrow 13.804$ \\
\hline
\end{tabular}

No: Protein numbering; Protein Name: Protein name; AH vs. NGM, GPDAC vs. NGM, LMGAC vs. NGM, LMGAC vs. GPDAC Represents the ratio of protein expression between the two stages; NGM: normal gastric mucosa, AH: atypical hyperplasia; GPDAC: gastric poorly differentiated adenocarcinoma; LMGAC: lymph nodes metastasis adenocarcinoma.

\section{Expression of IGHG 1 in gastric cancer and gastric cancer cells}

Firstly, the expression of IGHG1 protein in gastric cancer tissue chips (including 90 patients with gastric cancer) was detected by immunohistochemistry. The results showed that IGHG1 was mainly expressed in cell membrane and cytoplasm (Figure 1). The expression of IGHG1 in highly differentiated, moderately differentiated and poorly differentiated gastric adenocarcinoma tissues was higher than that in normal gastric mucosa tissues $(P<0.05)$ (Table 3). Next, we examined the expression of IGHG1 protein in gastric cancer SGC7901, MGC803, BGC823 cells and immortalized gastric mucosal epithelial GES-1 cells by western blot. The results showed that the expression of IGHG1 in SGC7901, MGC803 and BGC823 cells was significantly higher than that in GES- 1 cells $(P<0.05$, Figure 2A), and the expression level of SGC7901 cells was the highest. Therefore, we selected gastric cancer
SGC7901 cells for subsequent interference experiments.

Table 2. Quantitative proteomics to identify down-regulated proteins in different stages of gastric mucosal epithelial carcinoma

\begin{tabular}{|c|c|c|c|c|c|}
\hline No & Protein Name & $\begin{array}{l}\text { AH vs. } \\
\text { NGM }\end{array}$ & $\begin{array}{l}\text { GPDAC } \\
\text { vs. NGM }\end{array}$ & $\begin{array}{l}\text { LMGAC } \\
\text { vs. NGM }\end{array}$ & $\begin{array}{l}\text { LMGAC } \\
\text { vs. } \\
\text { GPDAC }\end{array}$ \\
\hline 1 & $\begin{array}{l}\text { Leucine-rich } \\
\text { repeat-containing protein } 59\end{array}$ & $\downarrow 0.655$ & $\downarrow 0.142$ & $\downarrow 0.273$ & $\downarrow 0.520$ \\
\hline 2 & Carboxyl ester lipase & $\downarrow 0.249$ & $\downarrow 0.191$ & $\downarrow 0.461$ & $\downarrow 0.413$ \\
\hline 3 & chymotrypsinogen B2 & $\downarrow 0.550$ & $\downarrow 0.236$ & $\downarrow 0.525$ & $\downarrow 0.449$ \\
\hline 4 & $\begin{array}{l}\text { Fibrinogen beta chain } \\
\text { precursor }\end{array}$ & $\downarrow 0.363$ & $\downarrow 0.240$ & $\downarrow 0.402$ & $\downarrow 0.597$ \\
\hline 5 & p180/ribosome receptor & $\downarrow 0.555$ & $\downarrow 0.242$ & $\downarrow 0.614$ & $\downarrow 0.394$ \\
\hline 6 & SERPINA3 & $\downarrow 0.242$ & $\downarrow 0.250$ & $\downarrow 0.154$ & $\downarrow 0.234$ \\
\hline 7 & Coronin-1A & $\downarrow 0.353$ & $\downarrow 0.281$ & $\downarrow 0.586$ & $\downarrow 0.167$ \\
\hline 8 & $\begin{array}{l}\text { PRSS3 Isoform A of Trypsin-3 } \\
\text { precursor }\end{array}$ & $\downarrow 0.273$ & $\downarrow 0.288$ & $\downarrow 0.143$ & $\uparrow 2.014$ \\
\hline 9 & RPL14 protein & $\downarrow 0.308$ & $\downarrow 0.322$ & $\downarrow 0.270$ & $\downarrow 0.172$ \\
\hline$\cdots$ & $\cdots$ & $\cdots$ & $\cdots$ & $\cdots$ & $\cdots$ \\
\hline 80 & $\begin{array}{l}\text { T-complex protein } 1 \text { subunit } \\
\text { beta }\end{array}$ & $\downarrow 0.319$ & $\downarrow 0.380$ & $\downarrow 0.013$ & $\downarrow 0.483$ \\
\hline 81 & $\begin{array}{l}\text { Isoform } 1 \text { of } \\
\text { Apoptosis-inducing factor } 1\end{array}$ & $\downarrow 0.643$ & $\downarrow 0.402$ & $\downarrow 0.570$ & $\downarrow 0.497$ \\
\hline 82 & RPS27A 79 kDa protein & $\downarrow 0.312$ & $\downarrow 0.449$ & $\downarrow 0.711$ & $\downarrow 0.631$ \\
\hline 83 & $\begin{array}{l}\text { HNRNPA1 Isoform A1-B of } \\
\text { Heterogeneous nuclear } \\
\text { ribonucleoprotein A1 }\end{array}$ & $\downarrow 0.619$ & $\downarrow 0.461$ & $\downarrow 0.474$ & $\downarrow 0.380$ \\
\hline 84 & $\begin{array}{l}\text { TSTA3 GDP-L-fucose } \\
\text { synthetase }\end{array}$ & $\downarrow 0.394$ & $\downarrow 0.479$ & $\downarrow 0.175$ & $\downarrow 0.325$ \\
\hline 85 & Calreticulin precursor & $\downarrow 0.291$ & $\downarrow 0.501$ & $\downarrow 0.425$ & $\downarrow 0.443$ \\
\hline 86 & $\begin{array}{l}\text { Heterogeneous nuclear } \\
\text { ribonucleoprotein U }\end{array}$ & $\downarrow 0.530$ & $\downarrow 0.530$ & $\downarrow 0.597$ & $\downarrow 0.213$ \\
\hline 87 & $\begin{array}{l}\text { LOC } 100130562 \text { hypothetical } \\
\text { protein isoform } 1\end{array}$ & $\downarrow 0.313$ & $\downarrow 0.545$ & $\downarrow 0.321$ & $\downarrow 0.380$ \\
\hline 88 & $\begin{array}{l}\text { SLC9A3R1 } \\
\text { Ezrin-radixin-moesin-binding } \\
\text { phosphoprotein } 50\end{array}$ & $\downarrow 0.406$ & $\downarrow 0.592$ & $\downarrow 0.149$ & $\downarrow 0.560$ \\
\hline 89 & $\begin{array}{l}\text { IMMT Isoform } 2 \text { of } \\
\text { Mitochondrial inner } \\
\text { membrane protein }\end{array}$ & $\downarrow 0.437$ & $\downarrow 0.608$ & $\downarrow 0.535$ & $\downarrow 0.209$ \\
\hline 90 & $\begin{array}{l}\text { Mitochondrial inner } \\
\text { membrane protein }\end{array}$ & $\downarrow 0.437$ & $\downarrow 0.608$ & $\downarrow 0.535$ & $\downarrow 0.209$ \\
\hline
\end{tabular}

No: Protein numbering; Protein Name: Protein name; AH vs. NGM, GPDAC vs. NGM, LMGAC vs. NGM, LMGAC vs. GPDAC Represents the ratio of protein expression between the two stages; NGM: normal gastric mucosa, AH: atypical hyperplasia; GPDAC: gastric poorly differentiated adenocarcinoma; LMGAC: lymph nodes metastasis adenocarcinoma.

\section{Establishment of pPLK-shRNA-IGHG 1 low expression cell line}

The pPLK-shRNA-IGHG1 three-interference plasmid and the negative control plasmid were transiently transfected into SGC7901 cells, and the non-transfected group was set. After 24 hours, the transfection efficiency was observed under a fluorescence microscope. The results showed that the transfection efficiency reached $80 \%-90 \%$, suggesting that the transfection efficiency was high and can be used in subsequent experiments (Figure 2B). Then, the total protein was extracted and the expression of IGHG1 protein in each group was detected by Western blot. The results showed that the pPLK-shRNAa-IGHG1 plasmid had an inhibition efficiency of $20 \%$, the pPLK-shRNAb-IGHG1 plasmid had an inhibition efficiency of $30 \%$, and the 
pPLK-shRNAc-IGHG1 plasmid had an inhibition interference plasmid was selected for subsequent efficiency of $70 \%$ (Figure 2C). Therefore, the shRNAc experiments.
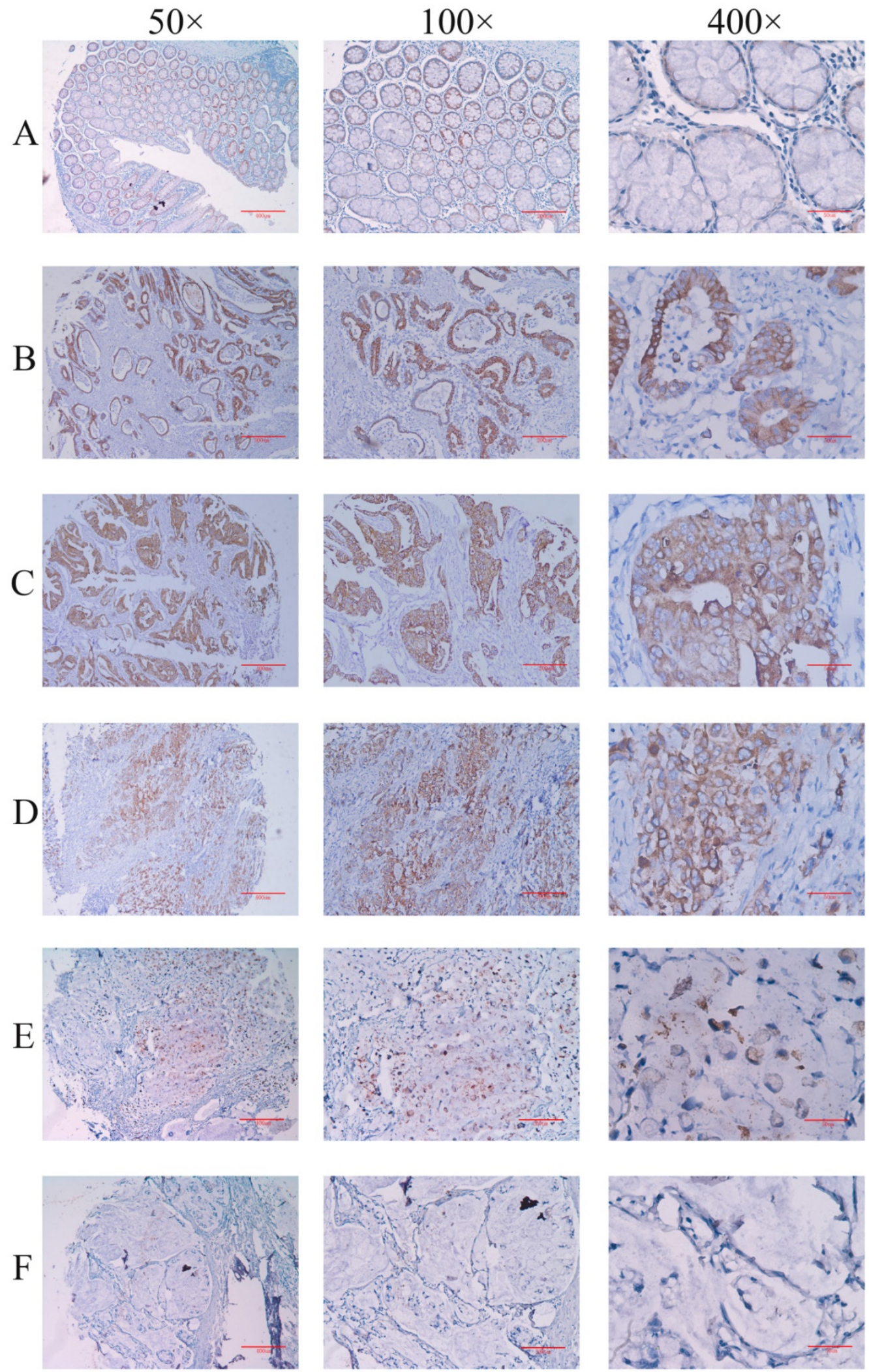

Figure 1. Immunohistochemical method for detecting the expression level of IGHGI in gastric cancer tissue microarray (A) Normal gastric mucosal tissue. (B) Highly differentiated adenocarcinoma tissue. (C) Moderately differentiated adenocarcinoma tissue. (D) Poorly differentiated adenocarcinoma tissue. (E) Signet ring cell carcinoma tissue. (F) Mucinous adenocarcinoma tissue. A-F: Original magnification: 50x, scale bar: $400 \mu \mathrm{m}$; Original magnification: $100 \times$, Scale bar: $200 \mu \mathrm{m}$; Original magnification: $400 \times$, scale bar: $50 \mu \mathrm{m}$. 
A

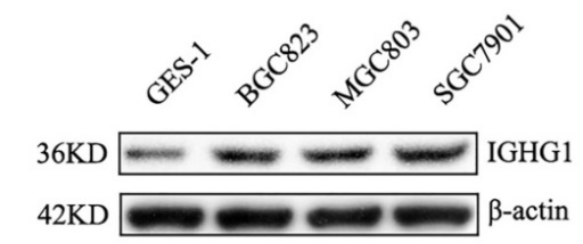

$\mathrm{B}$

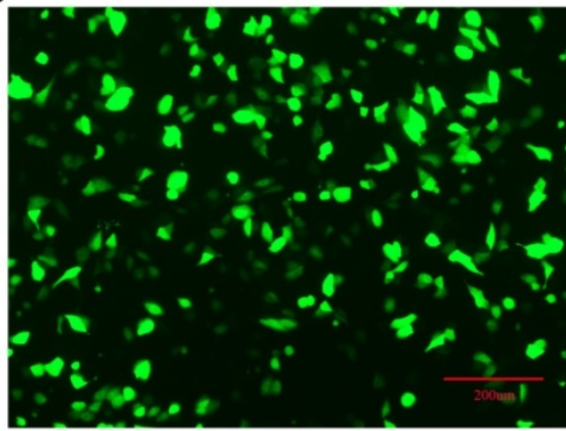

$\mathrm{C}$

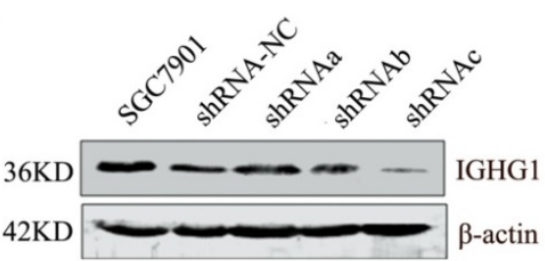

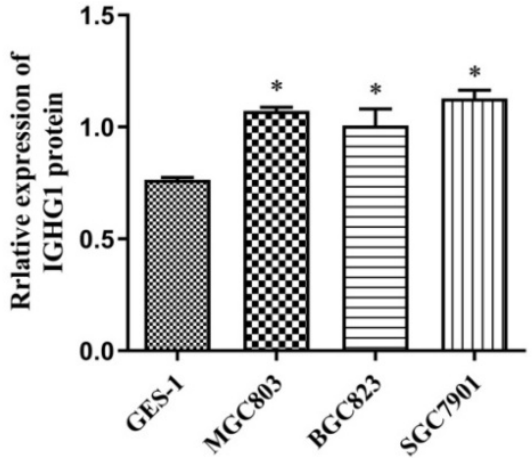
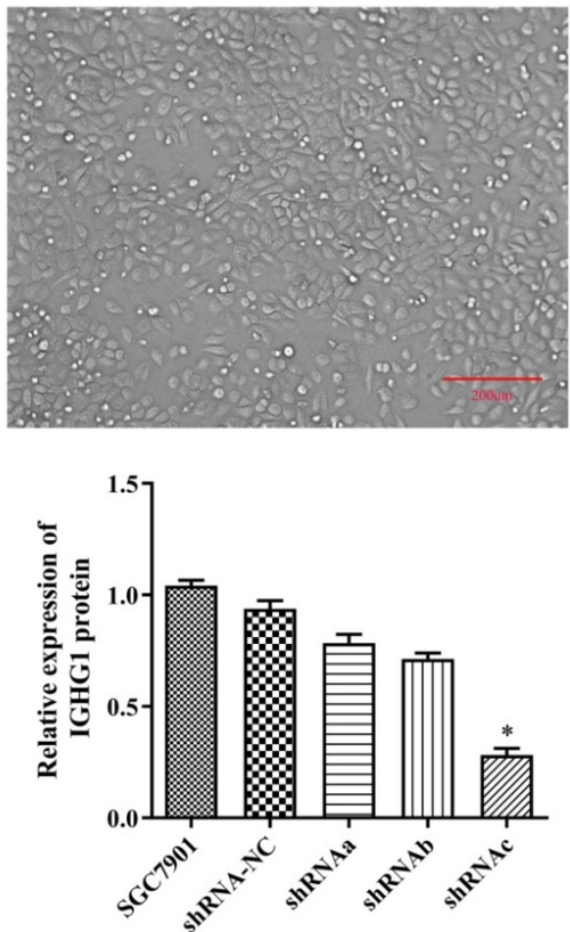

Figure 2. Detection of IGHGI expression level. (A) Western blotting was used to detect the expression of IGHG1 protein in different gastric cancer cell lines (BGC823, MGC803, SGC7901) and immortalized gastric mucosal cells GES-1. IGHGI was highly expressed in gastric cancer cell lines, and the highest expression was found in SGC7901 cells. (B) Fluorescent expression efficiency of gastric cancer cell SGC7901 after transient transfection of pPLK-shRNA-IGHG1. Original magnification, $100 \times$. Scale bar: $200 \mu$ m. (C) Western blotting was used to detect the expression of IGHGI protein after transfection of pPLK-shRNA-IGHGI (shRNA-NC, shRNAa, shRNAb, shRNAc), and the expression level of IGHG1 protein was the lowest in shRNAc group. *Compared with the control group, $P<0.05$.

Table 3. Expression analysis of IGHGI in gastric cancer

\begin{tabular}{llllll}
\hline Histological type & $\mathrm{n}$ & Negative & Positive & $\begin{array}{l}\text { IGHG1 [(n } \\
\%)]\end{array}$ & $P$ value \\
\hline normal & 70 & 35 & 35 & 50.00 & \\
highly differentiated & 20 & 4 & 16 & 80.00 & $0.033^{*}$ \\
$\begin{array}{l}\text { moderately } \\
\text { differentiated }\end{array}$ & 29 & 3 & 26 & 89.65 & $0.000^{*}$ \\
poorly differentiated & 21 & 2 & 19 & 90.48 & $0.002^{*}$ \\
signet ring cell & 9 & 2 & 7 & 77.77 & 0.223 \\
mucinous & 11 & 6 & 5 & 45.45 & 0.964 \\
\hline
\end{tabular}

${ }^{*}$ Compared with normal gastric mucosa tissue.

\section{Effect of low expression of IGHGI on proliferation of SGC790 I cells}

In order to investigate the effects of IGHG1 low expression on SGC7901 cell proliferation, the MTT experiment was first performed. The results showed that the proliferation ability of SGC7901 cells was decreased after low expression of IGHG1 $(P<0.05$, Figure $3 \mathrm{~A}-\mathrm{B})$. Then we conducted a plate colony formation experiment. The results showed that the cloning ability of SGC7901 cells was significantly decreased after low expression of IGHG1 $(P<0.05$, Figure $3 \mathrm{C}$ ). In conclusion, the results indicated that the proliferative capacity of SGC7901 cells was decreased after low expression of IGHG1, suggesting that IGHG1 may be involved in the proliferation of SGC7901 cells.

\section{Effect of low expression of IGHGI on migration and invasion of SGC7901 cells}

To understand the effect of low expression of IGHG1 on the migration of SGC7901 cells, we first performed a scratch test. The results showed that the 

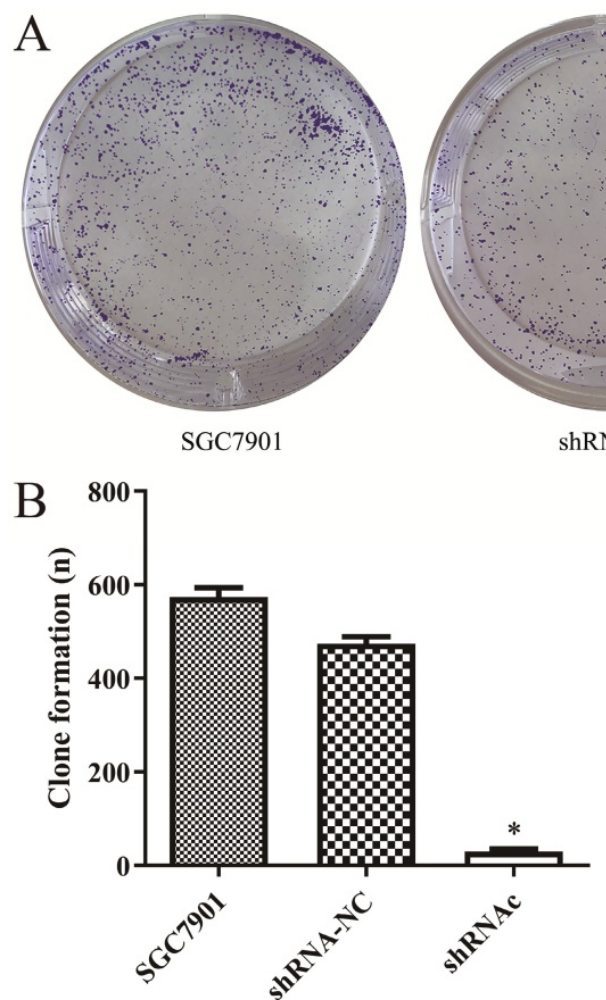

Figure 3. Low expression of IGHGI inhibits the growth and proliferation of gastric cancer SGC7901 cells. (A-C). Colony formation and MTT assays in shRNA-NC, and shRNAc groups were transfected into SGC7901 GC cell line. *Compared with the control group, $P<0.05$.

migration distance of SGC7901 cells was significantly lower than that of IGHG1 $(P<0.05$, Figure $4 \mathrm{~A}$ and $4 \mathrm{C})$. Then Transwell migrated the invasion experiment showed that the number of migration and invasion of SGC7901 cells was significantly decreased after low expression of IGHG1 $(P<0.05$, Figure $4 \mathrm{~B}$ and $4 \mathrm{D})$. In conclusion, the results showed that the migration and invasion ability of SGC7901 cells was decreased after low expression of IGHG1, suggesting that IGHG1 may be involved in the migration and invasion of SGC7901 cells.

\section{Effect of low expression of IGHGI on EMT-related molecules in SGC7901 cells}

To explore the effect of low expression of IGHG1 on EMT of SGC7901 cells and its mechanism of action. We used western blot to observe the effect of EMT-related proteins after low expression of IGHG1. The results showed that the expression of $\mathrm{N}$-cadherin, Vimentin and TGF- $\beta$ was decreased and the expression of E-cadherin was increased in SGC7901 cells after low expression of IGHG1 $(P<0.05$, Figure $5 \mathrm{~A})$. The results showed that the ability of EMT in SGC7901 cells was weakened after low expression of IGHG1.

To further understand the effect of low expression of IGHG1 on the TGF- $\beta$ signaling pathway. We used western blot to observe the changes of SMAD3 and P-SMAD3 after low expression of IGHG1. The results showed that the expression of P-SMAD3 in SGC7901 cells was decreased after low expression of IGHG1 $(P<0.05$, Figure 5B), but the expression of SMAD3 was not significantly changed $(P>0.05)$. The results indicated that IGHG1 induced EMT in SGC7901 cells by regulating the TGF- $\beta$ /SMAD3 pathway.

\section{Discussion}

Gastric cancer is one of the malignant tumors with the highest morbidity and mortality in the world [1]. The occurrence and development of gastric cancer is a complex pathological process involving multiple stages and multiple factors.

Quantitative proteomic analyses can provide information for the search of potential biomarkers [10]. At present, laser capture microdissection (LCM) is one of the best methods to purify tissue [11]. Isobaric tags for relative and absolute quantification (iTRAQ) is widely used for differential expression and quantitative detection [12]. The quadrupole time-of-flight tandem mass spectrometer (Q-TOF MS/MS) has high accuracy, high sensitivity and high resolution, which is conducive to the determination of extreme molecular weight, extreme $\mathrm{PH}$ and low abundance proteins [13]. According to iTRAQ technology and 2D LCMS/MS, 243 differentially expressed proteins related to gastric cancer were preliminarily screened and identified, including IGHG1. It provides abundant material and experimental basis for search proteins in early diagnosis of gastric cancer.

The IGHG1 gene is located on chromosome 14, which is a protein-coding gene and is closely related to the occurrence and development of tumors. Immunoglobulin was traditionally thought to be produced by lymphocytes and plasma cells. Heavy and light chains of IgG have been detected in the cytoplasm of many human cancer cell lines [14]. The study found that IGHG1 was highly expressed in tumors such as ovarian cancer, and participates in pathological processes such as EMT, proliferation, apoptosis resistance, immune escape and metastasis 

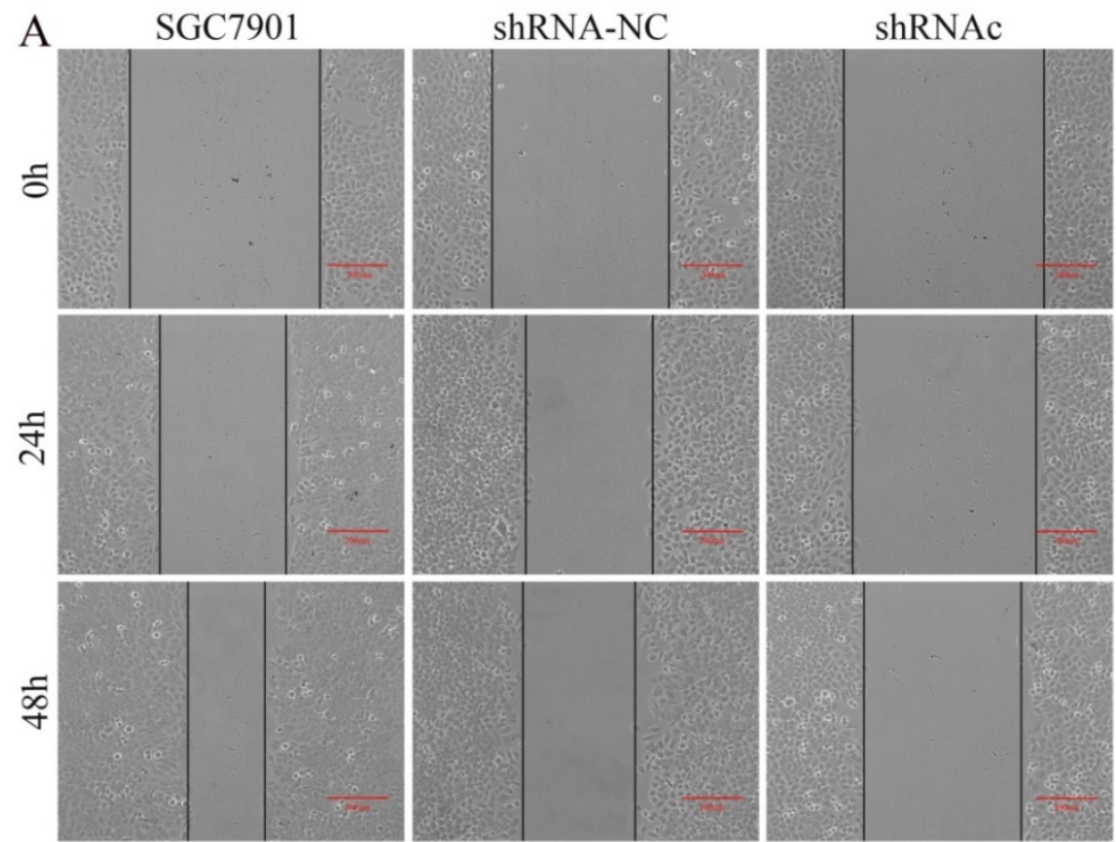

$\mathrm{B}$
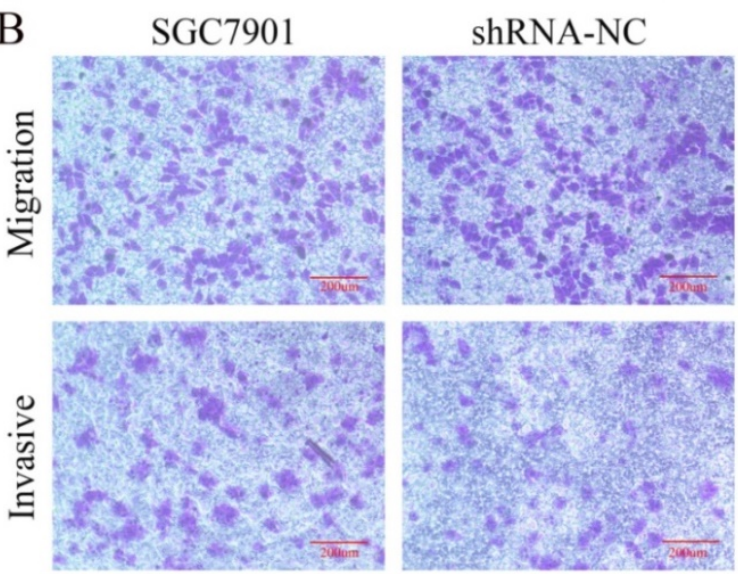

$\mathrm{C}$

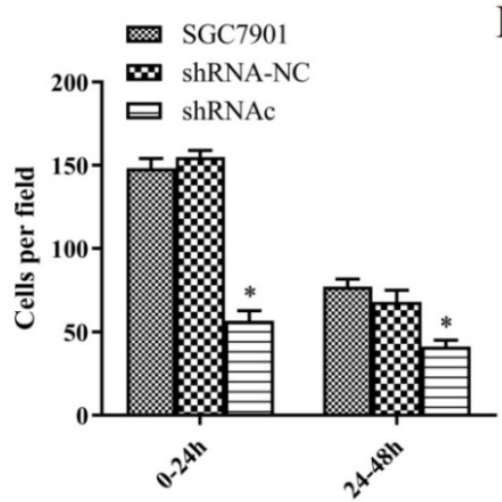

$\mathrm{D}$

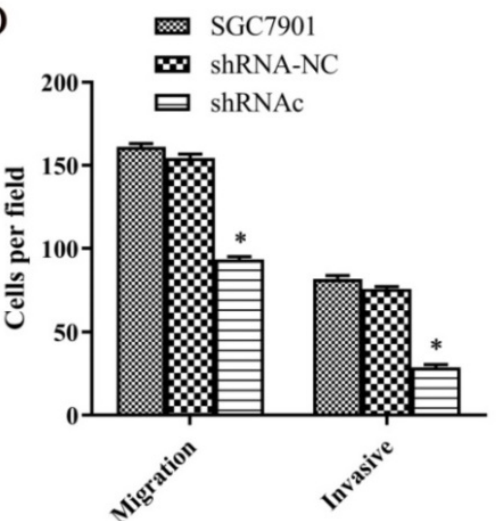

Figure 4. Low expression of IGHGI inhibits migration and invasion of gastric cancer cell line SGC7901. SGC7901 cells were transfected with shRNA-NC and shRNAc, respectively. (A) Wound healing assay in the shRNA-NC, and shRNAc groups were transfected into SGC7901 GC cell line. (C) Migration index in the shRNA-NC, and shRNAc groups. Original magnification, 100×. scale bar: $200 \mu \mathrm{m}$. (B, D) Transwell migration and invasion assays in the shRNA-NC, and shRNAc groups. Original magnification, 100x. scale bar: $200 \mu \mathrm{m}$ Results are expressed as the mean \pm SD with significance at $* P<0.05$. *Compared with the control group, $P<0.05$.

of tumor cells [6, 7]. Studies have also found that IGHG1 was significantly more expressed in pancreatic cancer tissues than in non-cancer tissues,
IGHG1 downregulates the cytotoxic effects of NK cells by inhibiting the antigen-dependent cytotoxic function, leading to proliferation and immune escape of pancreatic cancer cells [15]. In current study, low expression of IGHG1 can inhibit the growth and induce apoptosis of prostate cancer cells [8]. Its expression and mechanism of action in gastric cancer remains unclear. Based on previous studies, we found that IGHG1 was highly expressed in gastric cancer, participated in the proliferation, migration and invasion of SGC7901 cells and affects EMT of SGC7901 cells.

A large number of studies have found that the occurrence of EMT biological behavior plays an important role in the metastasis of malignant tumors [16-18]. In gastric cancer, many signaling pathways were involved in the regulation of EMT, and signaling pathways such as PI3K/AKT [19], MEK/ERK [20], and $\quad W N T / \beta-$ Catenin [21], especially TGF- $\beta$ / SMAD [22, 23] play important roles. Tumor cells transform from epithelial cells to stromal cells stimulated by TGF- $\beta$ signaling was mediated by the SMAD pathway [24, 25]. Members of the SMAD protein family are transcription factors that transmit TGF- $\beta$ stimulation signals from cell membranes to the nucleus $[26,27]$. In our experiments, the expression of EMT signaling pathway protein TGF- $\beta$ was decreased in SGC7901 cells when IGHG1 was poorly expressed, indicating that IGHG1 may promote the migration and invasion of gastric cancer cells through the TGF- $\beta /$ SMAD signaling pathway. We found that the expression of P-Smad3 was significantly reduced after IGHG1 was poorly expressed, while the expression of SMAD3 was not significantly changed, which indicated that IGHG1 induced EMT in SGC7901 cells and promoted cell migration and invasion by regulating the activation of TGF- $\beta$ /SMAD3 signaling pathway. Although IGHG1 
A

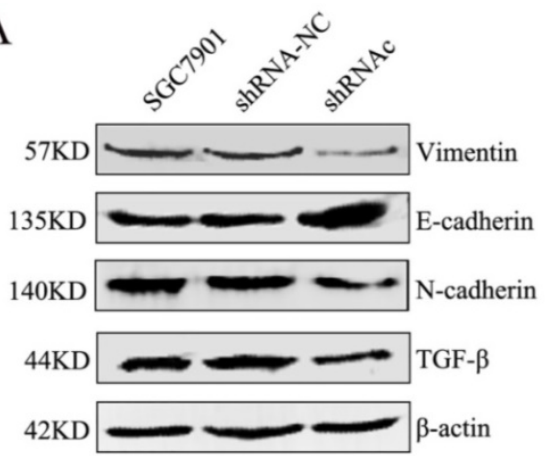

B

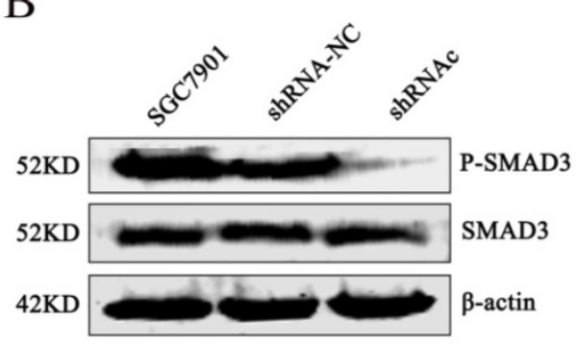

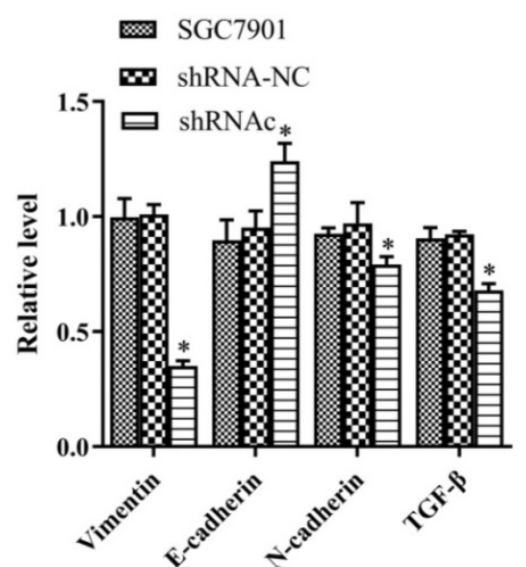

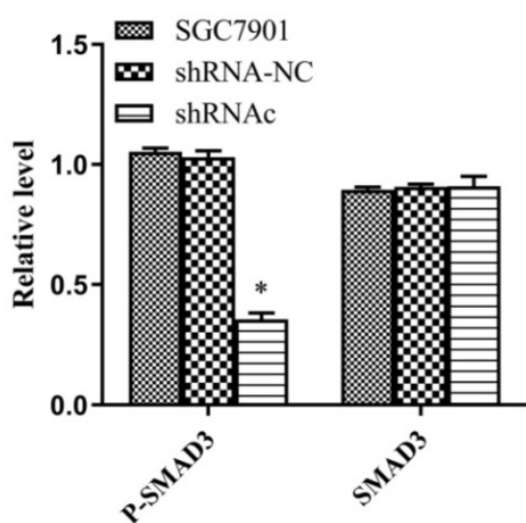

Figure 5. Low expression of IGHG I inhibits EMT (A) Western blotting shows changes in the expression levels of EMT related proteins N-cadherin, E-cadherin, Vimentin and TGF- $\beta$ in the shRNA-NC, and shRNAc groups. (B) Western blotting shows changes in the expression levels of SMAD3 and P-SMAD3 in the shRNA-NC, and shRNAC groups. Results are expressed as the mean \pm SD with significance at $* P<0.05$.

interfered to reverse EMT occurrence in SGC7901 cells, there are still some shortcomings in this study. For example, this study only carried out follow-up analysis at the level of cell interference, and did not carry out in vivo experiments, which need to be verified by further experiments.

\section{Conclusions}

This study found that IGHG1 induced cell migration and invasion by inducing EMT in SGC7901 cells by modulating the activation of the TGF- $\beta$ /SMAD3 signaling pathway. The molecular mechanism of IGHG1 affecting EMT in gastric cancer SGC7901 cells was preliminarily confirmed, which provided experimental and theoretical basis for elucidating the molecular mechanism of gastric cancer.

\section{Acknowledgements}

This work was supported by Hunan Provincial Groundbreaking Platform Open Fund of University of China (NO. 18K076), Doctoral Research Fund of University of South China (NO. 2016XQD21), University of South China and Horizontal Cooperation Project of Yueyang Maternal and Child
Health Hospital (NO. 2018KHX43), Hunan Provincial Health Department Project (NO. B2019119), Hunan Science and Technology Innovation Project (NO. 2018SK51601), Natural Science Foundation of Hunan Province (NO. 20JJ4083) and Hunan Postgraduate Science and Technology Innovation Project (NO. CX20200959).

\section{Competing Interests}

The authors have declared that no competing interest exists.

\section{References}

1. Bray F, Ferlay J, Soerjomataram I, Siegel RL, Torre LA, Jemal A. Global cancer statistics 2018: GLOBOCAN estimates of incidence and mortality worldwide for 36 cancers in 185 countries. CA Cancer J Clin. 2018; 68: 394-424.

2. Chen $\mathrm{W}$, Zheng $\mathrm{R}$, Baade PD, Zhang S, Zeng H, Bray $\mathrm{F}$, et al. Cancer statistics in China, 2015. CA Cancer J Clin. 2016; 66: 115-32.

Li X, Ni R, Chen J, Liu Z, Xiao M, Jiang F, et al. The presence of IGHG1 in human pancreatic carcinomas is associated with immune evasion mechanisms. Pancreas. 2011; 40: 753-61.

4. Stickler MM, Reddy A, Xiong JM, Hinton PR, DuBridge R, Harding FA. The human G1m1 allotype associates with CD4+ T-cell responsiveness to a highly conserved IgG1 constant region peptide and confers an asparaginyl endopeptidase cleavage site. Genes Immun. 2011; 12: 213-21.

5. Qiu Y, Korteweg C, Chen Z, Li J, Luo J, Huang $G$, et al. Immunoglobulin $G$ expression and its colocalization with complement proteins in papillary thyroid cancer. Mod Pathol. 2012; 25: 36-45.

6. Qian J, Ji F, Ye X, Cheng H, Ma R, Chang X, et al. IGHG1 promotes motility likely through epithelial-mesenchymal transition in ovarian cancer. Chin J Cancer Res. 2018; 30: 282-90.

7. Ji F, Chang X, Liu C, Meng L, Qu L, Wu J, et al. Prognostic value and characterization of the ovarian cancer-specific antigen CA166-9. Int J Oncol. 2015; 47: 1405-15.

8. Pan B, Zheng S, Liu C, Xu Y. Suppression of IGHG1 gene expression by siRNA leads to growth inhibition and apoptosis induction in human prostate cancer cell. Mol Biol Rep. 2013; 40: 27-33.

9. Xu Y, Chen B, Zheng S, Wen Y, Xu A, Xu K, et al. IgG silencing induces apoptosis and suppresses proliferation, migration and invasion in LNCaP prostate cancer cells. Cell Mol Biol Lett. 2016; 21: 27.

10. Kang C, Lee Y, Lee JE. Recent advances in mass spectrometry-based proteomics of gastric cancer. World J Gastroenterol. 2016; 22: 8283-93.

11. Aguilar-Bravo B, Sancho-Bru P. Laser capture microdissection: techniques and applications in liver diseases. Hepatology International. 2019; 13: 138-47.

12. Moulder R, Bhosale SD, Goodlett DR, Lahesmaa R. Analysis of the plasma proteome using iTRAQ and TMT-based Isobaric labeling. Mass Spectrom Rev. 2018; 37: 583-606.

13. Xie C, Zhong DF, Yu KT, Chen XY. Recent advances in metabolite identification and quantitative bioanalysis by LC-Q-TOF MS. Bioanalysis. 2012; 4: 937-59.

14. Chen $\mathrm{Z}, \mathrm{Gu} J$. Immunoglobulin $\mathrm{G}$ expression in carcinomas and cancer cell lines. FASEB J. 2007; 21: 2931-8.

15. Li XY, Ni RZ, Chen JH, Liu ZX, Xiao MB, Jiang F, et al. The Presence of IGHG1 in Human Pancreatic Carcinomas Is Associated With Immune Evasion Mechanisms. Pancreas. 2011; 40: 753-61.

16. Xie L, Jiang $\mathrm{T}$, Cheng $\mathrm{A}$, Zhang $\mathrm{T}$, Huang $\mathrm{P}, \mathrm{Li}$, et al. MiR-597 Targeting 14-3-3sigma Enhances Cellular Invasion and EMT in Nasopharyngeal Carcinoma Cells. Curr Mol Pharmacol. 2019; 12: 105-14.

17. Hung JJ, Kao YS, Huang CH, Hsu WH. Overexpression of Aiolos promotes epithelial-mesenchymal transition and cancer stem cell-like properties in lung cancer cells. Sci Rep. 2019; 9: 2991

18. Shi DM, Li LX, Bian XY, Shi XJ, Lu LL, Zhou HX, et al. miR-296-5p suppresses EMT of hepatocellular carcinoma via attenuating NRG1/ERBB2/ERBB3 signaling. J Exp Clin Cancer Res. 2018; 37: 294. 
19. Wei R, Xiao Y, Song Y, Yuan H, Luo J, Xu W. FAT4 regulates the EMT and autophagy in colorectal cancer cells in part via the PI3K-AKT signaling axis. J Exp Clin Cancer Res. 2019; 38: 112.

20. Wang Y, Gu J, Lin X, Yan W, Yang W, Wu G. IncRNA BANCR promotes EMT in PTC via the Raf/MEK/ERK signaling pathway. Oncol Lett. 2018; 15: $5865-70$.

21. Liu F, Xia Z, Zhang M, Ding J, Feng Y, Wu J, et al. SMARCAD1 Promotes Pancreatic Cancer Cell Growth and Metastasis through Wnt/beta-cateninMediated EMT. Int J Biol Sci. 2019; 15: 636-46.

22. Ooshima A, Park J, Kim SJ. Phosphorylation status at Smad3 linker region modulates transforming growth factor-beta-induced epithelial-mesenchymal transition and cancer progression. Cancer Sci. 2019; 110: 481-8.

23. Tanaka T, Goto K, Iino M. Sec8 modulates TGF-beta induced EMT by controlling N-cadherin via regulation of Smad3/4. Cell Signal. 2017; 29: 115-26.

24. Zhang $X$, Zhang $P$, Shao M, Zang $X$, Zhang J, Mao F, et al. SALL4 activates TGF-beta/SMAD signaling pathway to induce EMT and promote gastric cancer metastasis. Cancer Manag Res. 2018; 10: 4459-70.

25. Yao Y, Chen R, Wang G, Zhang Y, Liu F. Exosomes derived from mesenchymal stem cells reverse EMT via TGF-beta1/Smad pathway and promote repair of damaged endometrium. Stem Cell Res Ther. 2019; 10: 225.

26. Sakai S, Ohhata T, Kitagawa K, Uchida C, Aoshima T, Niida H, et al. Long Noncoding RNA ELIT-1 Acts as a Smad3 Cofactor to Facilitate TGFbeta/Smad Signaling and Promote Epithelial-Mesenchymal Transition. Cancer Res. 2019; 79: 2821-38.

27. Tu S, Huang W, Huang C, Luo Z, Yan X. Contextual Regulation of TGF-beta Signaling in Liver Cancer. Cells. 2019; 8: 1235. 